\title{
Building stable stellar systems with $\rho$ as the only datum
}

\section{D. Carpintero}

Facultad de Ciencias Astronómicas y Geofísicas, UNLP, Argentina

Instituto de Astrofísica de La Plata, CONICET-UNLP, Argentina

\begin{abstract}
Schwarzschild's method to set up a model galaxy is most useful when the distribution in velocity space is unknown. Nevertheless, one has to know beforehand which kind of orbits are spawned by the potential of the model. Moreover, although the system thus generated is in equilibrium, it is not necessarily stable. Here, we present a new method that allows to build up a stable stellar system without any previous knowledge of its distribution in velocity space.
\end{abstract}

Keywords. methods: numerical, galaxies: kinematics and dynamics

The full poster (in pdf format) is available at http://www.astro.iag.usp.br/〜iaus266/Posters/pCarpintero.pdf. 